\title{
Study on Efficient Fused Deposition Modelling of Thermoplastic Polyurethane Inflatable Wall Features for Airtightness
}

\author{
Mo CHEN ${ }^{\mathrm{a}}$, Qinglei JI ${ }^{\mathrm{a}, \mathrm{b}}$, Xiran $\mathrm{ZHANG}^{\mathrm{b}}$, Lei FENG ${ }^{\mathrm{b}}$, \\ $\mathrm{Xi}$ Vincent WANG ${ }^{\mathrm{a}}$ and Lihui WANG ${ }^{\mathrm{a}, 1}$ \\ ${ }^{a}$ Department of Production Engineering, KTH Royal Institute of Technology, Sweden \\ ${ }^{\mathrm{b}}$ Department of Machine Design, KTH Royal Institute of Technology, Sweden
}

\begin{abstract}
The thermoplastic polyurethane (TPU) material is an elastomer that can be used for inflatable products. Fused deposition modelling (FDM) is a widely used additive manufacturing process for TPU material due to the capability of generating complex structures with low cost. However, TPU is soft and thus difficult to be extruded as continuously and uniformly as hard materials such as polylactide by FDM. Inappropriate extruder structure and speed settings can lead to filament buckling problem, resulting in poor material filling quality, long printing time and low printing success rate. This paper aims at improving the FDM printing efficiency of TPU inflatable products by adding lateral support to the filament and finding out the appropriate speed ranges for different wall features and thicknesses. Firstly, a filament guide sheet is designed as being inserted into the gap between the drive gears and the bottom frame of the gear chamber in order to prevent the soft TPU filament from buckling. Secondly, inflatable product wall features are classified into floors, roofs and sidewalls and experiment for finding the relationship between printing speed and airtightness is carried out. In order to verify the proposed solution, wall features are printed and the material fillings obtained under different printing speeds are compared by measuring the airtightness of the wall features. Results show that the proposed filament guide sheet mitigates filament buckling, and the speed range that meets the airtightness requirement can be found for various wall features and thicknesses. In summary, the sealing of the filament feeding channel between the drive gears and the nozzle, as well as the speed optimisation according to product features, are essential for the efficient printing of TPU inflatable products.
\end{abstract}

Keywords. Thermoplastic polyurethane, 3D printing, fused deposition modelling, filament extrusion

\section{Introduction}

Inflatable products, such as pneumatically driven soft robots, pneumatic tires and balloon catheters, are widely used due to their advantages of adaptive shapes and impact energy absorption. Soft materials, such as thermoplastic polyurethane (TPU), in inflatable products act as absorbers or buffers during collision and thus it is usually safer for inflatable objects to touch human body during interaction compared to products with rigid shells. With a fixed or controllable amount of air inside an

\footnotetext{
${ }^{1}$ Corresponding Author. lihuiw@kth.se
} 
inflatable object, the volume and pressure of the inflatable object can be changed for the interaction between the inflatable object and another object on specified surfaces. For example, with a controllable air pressure, an inflatable object can change its shape during the movement inside a curved narrow tunnel to improve its accessibility. Pneumatically driven soft robots are able to perform difficult actions like bending, wrinkling and twisting which cannot be done by the rigid robot due to the limitation of the degree of freedom [1] as well as manipulate fragile objects with complex surfaces without applying large force to damage the objects.

Inflatable products are generally made through joining and sealing materials by gluing, sewing or high-frequency welding [2]. Such sealing methods are simple and fast and have been used in fabrication of inflatable robotic arms [3] and soft textile inflatable actuators [4] recently. However, it becomes challenging when complex fine structure is to be formed through conventional gluing, sewing and welding. 3D moulding offers another solution for fast mass production of inflatable products, but the demoulding step has limited the possible structures fabricated by 3D moulding [5]. For production of a few individualised inflatable products, the material and time costs for mould manufacturing are not negligible. Moreover, it is generally not a preferable choice to make multi-material products through moulding since it is difficult to precisely set the positions of various materials in a mould chamber. $2 \mathrm{D}$ moulding and full lithography have been reported as feasible methods for producing micro inflatable actuators [5]. In recent years, $3 \mathrm{D}$ printing has become a popular way of manufacturing small-scale individualised products including inflatable products due to its voxel-wise additive style which provides great flexibility to design of functional delicate products. Typical examples that have been 3D printed include soft robotic fingers [6], soft grippers [7][8], robotic wrist sleeves [9] and linear vacuum actuators [10]. Many of the 3D printed inflatable products are fabricated using FDM (fused deposition modelling) due to its simplicity with low cost.

Airtightness is one of the most important specifications for air inflatable products. If there is air leakage with an inflatable product, its pressure and/or volume accuracy will deteriorate and the product could even lose its functionality. Wang et al. tested FDM printing of peristaltic microfluidic systems and proposed to use printing speed of $5 \mathrm{~mm} / \mathrm{s}$ and wall thickness of $1 \mathrm{~mm}$ to avoid gaps which would otherwise lead to liquid leakage [11]. Although various inflatable products have been printed, the relationship between product airtightness and printing parameters considering product features has not been discussed in detail in existing publications. In this paper, the FDM printability improvement of TPU, which is widely used for inflatable products [12], as well as the airtightness of various printed features will be discussed.

\section{Improvement of FDM printability for TPU}

In FDM printing, the material is deposited layer by layer through feeding the filament to the hot end by a pair of gears. Filament is melted at the hot end and the filament viscosity will drop in order to let the filament be sheared and extruded out of the nozzle. The resistance applied by the hot end and the driving force applied by the gears on the filament together lead to buckling of the filament segment between the gears and the hot end, as shown in Figure 1. If the hot end temperature is not high enough, the filament will not be able to melted thoroughly, the viscosity of the filament will remain high and thus high shear force will be needed from gear and hot end to feed the 
filament through the nozzle, which will raise the possibility of filament buckling and even lead to filament warping around the gears.

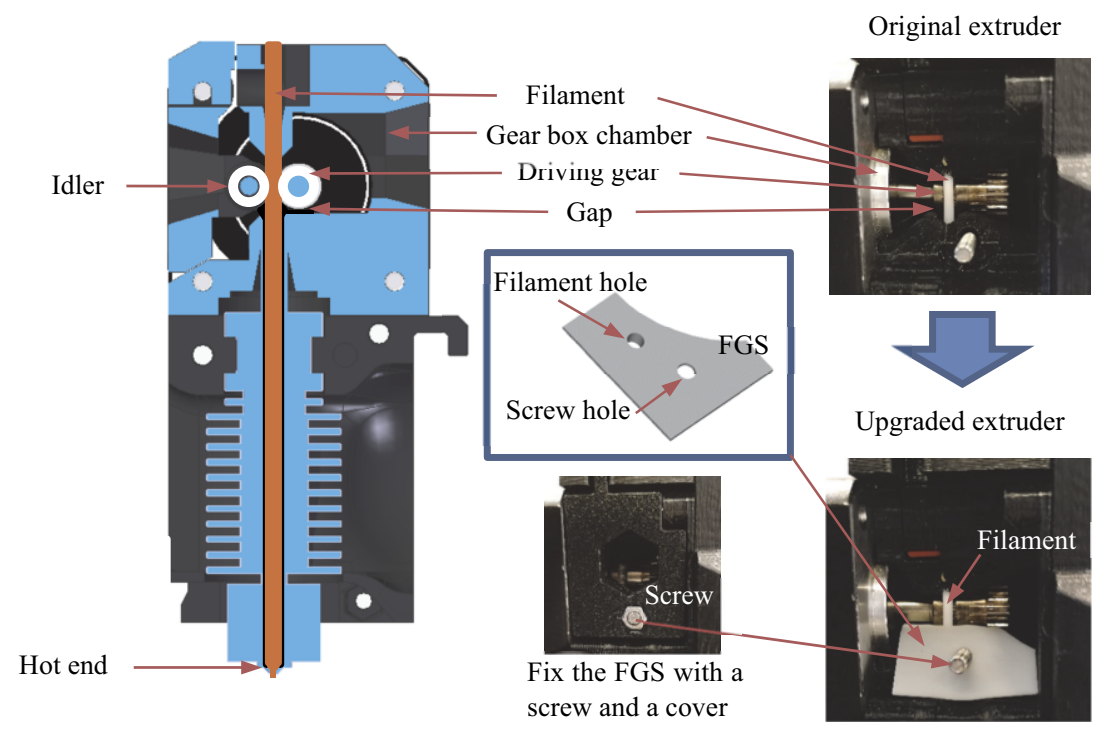

Figure 1. Original extruder upgraded with a filament guide sheet (FGS)

The filament buckling problem can be explained by the Euler's critical buckling load equation [13]:

$$
F_{\mathrm{cr}}=\frac{\pi^{2} E I}{(k L)^{2}}
$$

Where $F_{\mathrm{cr}}$ is the axial critical force above which filament buckling will happen, $E$ is the modulus of elasticity of the filament, $L$ is the unsupported length of the filament, $I$ is the area moment of inertia of the filament's cross section and $k$ is a constant related to the filament segment's end supports. For soft filaments with low modulus of elasticity, the unsupported length of the filament shall also be kept low to be able to sustain high extrusion force from the gears. As shown in Figure 1, the gap corresponding to the unsupported filament segment lies between the gears and the chamber bottom of the gear box. For soft materials such as TPU, the buckling problem is more serious than typical stiff printing materials. As a result, it is difficult to extrude TPU as continuously and uniformly as stiff materials. This will lead to either over-extrusion or underextrusion which form undesirable gaps during printing.

If a lateral support can be added to the gap for the filament, the unsupported length $L$ can be reduced and thus the critical extrusion force without causing filament buckling will increase. In this paper, an elastic filament guide sheet (FGS) is designed as being inserted into the gap to support the filament from lateral. The hole diameter for filament requires careful design, since an undersized hole will cause friction between the filament and the guide sheet while an oversized hole will give poor support to the filament. For the TPU filament with diameter of $1.75 \mathrm{~mm}$ used in this paper, we choose $1.9 \pm 0.1 \mathrm{~mm}$ as the filament hole diameter for FGS. As will be shown in Section 3.2.1, 
the maximum volumetric speed for printing TPU can reach at least $3.7 \mathrm{~mm}^{3} / \mathrm{s}$ (infill speed of $80 \mathrm{~mm} / \mathrm{s}$ with extrusion multiplier of 1.1) by using the FGS.

\section{FDM printing for airtightness}

To print an inflatable product, the 3D model of the product is imported into a slicer programme first, and G-code will be generated by the slicer in terms of parameters that are set by users. The airtightness of a printed inflatable product is related to both the features of the product and the FDM parameters set by the slicer.

\subsection{Inflatable wall features}

In FDM printing, the layers of a print are determined by the direction of placing the designed model. There is at least one chamber with walls in an inflatable product. We classify the wall features of an inflatable product into three types as shown in Figure 2:

1. Floor. The floor is the bottom of a chamber and is least affected by gravity. A floor with a horizontal inner surface has layers whose normal directions are parallel to the air pressure's direction.

2. Sidewall. A sidewall with a vertical inner surface has layers whose normal directions are perpendicular to the air pressure's direction. It is usually challenging to print a tall thin sidewall with a thickness lower than $0.5 \mathrm{~mm}$ since the gravity of higher layers tends to press the lower layers and lead to buckling of the sidewall.

3. Roof. Similar to floors, a roof with a horizontal inner surface has layers whose normal directions are parallel to the air pressure's direction. However, if an inflatable product is printed without support materials, the layer forming of the roofs will be based on bridges and greatly affected by gravity.

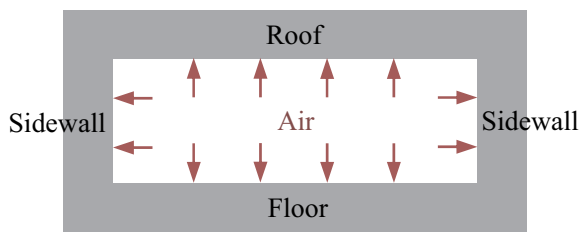

Figure 2. Classification of inflatable wall features

In order to simplify the discussion, this paper covers only the horizontal floor, vertical sidewall and horizontal roof and the inner and outer surfaces of a wall are parallel to each other. Walls with non-uniform thickness and slope surfaces are beyond the scope of this paper.

\subsection{FDM parameters related to airtightness}

FDM parameters can be tuned in the slicer programme to change the tool path and the printer's hardware settings (e.g., temperatures and motor speeds). In this paper, 
PrusaSlicer 2.1.0 is used as the slicer. The key parameters that affect the airtightness of a print are given below.

1. Printing speed. The printing speed is directly related to the total printing time. In the slicer programme the printing speed setting is usually divided into multiple parameters for different types of features such as perimeters, supports, bridges and infills. Provided that any part of the filament can be extruded within the desired temperature range without under- or over-extrusion which can lead to gaps on the print's surface, it is usually preferred to set the printing speed as high as possible for large area printing. If the printing area of a layer is too small, the deposited layer will be heated up again soon by the hot end and may collapse due to the long cooling time.

2. Temperature parameters, including extruder temperature, bed temperature and cooling fan speed. As mentioned in Section 1, the extruder temperature affects the viscosity of the filament near the hot end. Higher printing speed requires higher extruder temperature to avoid under-extrusion (i.e., to ensure that the filament material is melted to be with a viscosity that allows the filament to be sheared out from the nozzle). However, an over-heated hot end will also warm up the deposited material and modify the near feature that has been formed in earlier extrusion. The bed temperature and cooling fan speed can also affect the temperature field at the printing area and the flow and solidification of the material which has been extruded.

3. Volumetric speed parameters, including extrusion multiplier and extrusion width. The volumetric speed of material extruded can be tuned by changing the extrusion multiplier and width. Over-large volumetric speed will lead to over-extrusion while over-small volumetric speed will lead to under-extrusion.

In this paper, we fix the temperature and volumetric speed parameters and study how the printing speed affects the airtightness of inflatable product.

\section{Experiment}

\subsection{Experimental setup}

The experimental platform for the wall features includes two parts: the FDM printer and the airtightness testing device. The floors, sidewalls and roofs are first printed by a Prusa i3 MK3 printer (Figure 3) and then mounted in the airtightness test device (Figure 4) to measure the pressure drop.

In order to fix the printed wall firmly into the test device while considering the printability of the walls, the wall samples are designed in various shapes. Each floor or roof wall is designed as a square sheet surrounded by a frame with width of $1.8 \mathrm{~mm}$. The edge length of the sheet is $20.2 \mathrm{~mm}$ excluding the frame and the height of the sheet is the same as the wall thickness to be tested. Each sidewall sample is printed as a hollow cylinder (inner diameter $20 \mathrm{~mm}$, and height $32 \mathrm{~mm}$ with the wall thickness to be tested) and then cut into two samples. The filament for printing inflatable products is TPU-based NinjaTek NinjaFlex. The FDM slicing and printing parameters are given in Table 1. 


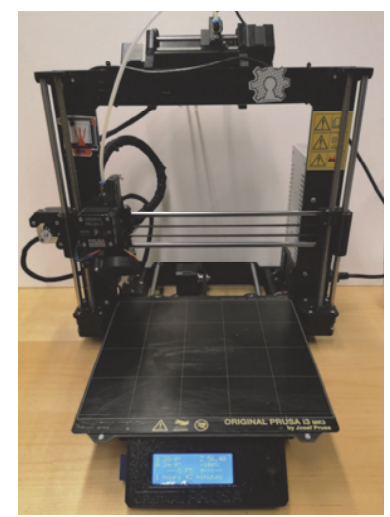

Figure 3. The Prusa i3 MK3 printer for printing wall features

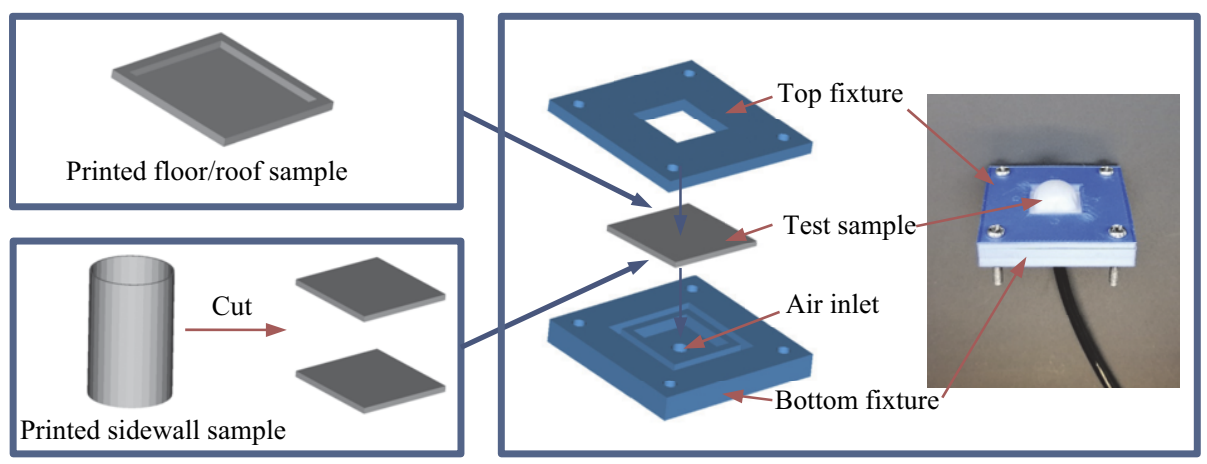

Figure 4. Airtightness test for printed wall samples

Table 1. Parameters for printing wall features.

\begin{tabular}{cc}
\hline Parameter name & Value \\
\hline Layer height & $0.1(\mathrm{~mm})$ \\
Infill speed & Floor: $20,40,60,80(\mathrm{~mm} / \mathrm{s})$ \\
Extrusion width & Sidewall and roof: $10,20,30,40(\mathrm{~mm} / \mathrm{s})$ \\
Extrusion multiplier & $0.45(\mathrm{~mm})$ \\
Extruder temperature & 1.1 \\
Bed temperature & $245\left({ }^{\circ} \mathrm{C}\right)$ \\
Fill density & $40\left({ }^{\circ} \mathrm{C}\right)$ \\
Fill pattern & $100 \%$ \\
Cooling fan speed & Rectilinear \\
\end{tabular}

In the airtightness test, the initial air pressure is set to 1.80 bar. When the valve is turned on, the chamber with the printed wall sample as one of the walls is inflated and the air pressure at the air inlet of the test device is measured by a Festo SPAU-P10R pressure sensor. The pressure drop value, which is the difference between the initial and measured air pressure, indicates the airtightness of a printed wall. 


\subsection{Experimental results}

\subsubsection{Printability}

Figure 5 compares the filament feeding in the printing of a floor sample with the thickness of $0.5 \mathrm{~mm}$ with and without FGS. The idler is disassembled from the extruder to show the filament. Filament buckling can occur even at the low speed of $20 \mathrm{~mm} / \mathrm{s}$ without lateral support by FGS (Figure 5 (a)). With FGS, the speed of $20 \mathrm{~mm} / \mathrm{s}$ (Figure 5 (b)) and even $80 \mathrm{~mm} / \mathrm{s}$ (Figure 5 (c)) can be used without causing filament buckling. Therefore, the printability of TPU can be improved by FGS.

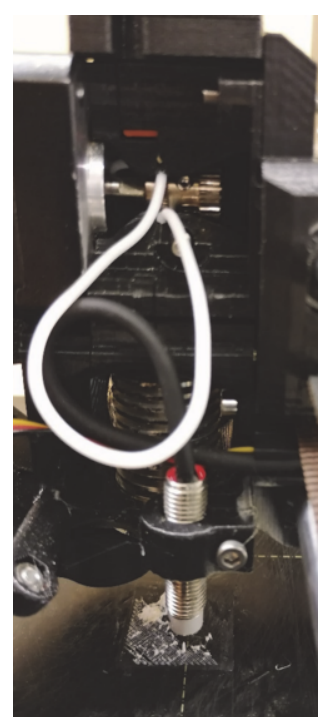

(a) $20 \mathrm{~mm} / \mathrm{s}$ without FGS

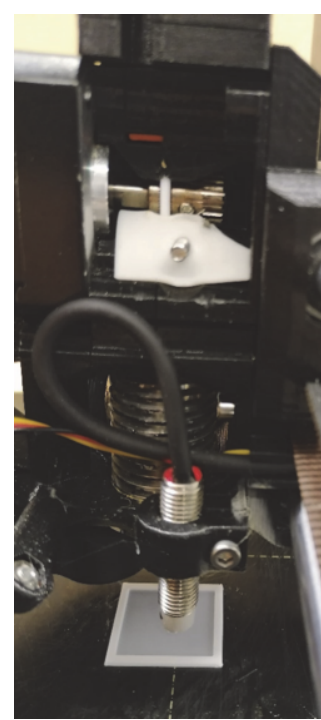

(b) $20 \mathrm{~mm} / \mathrm{s}$ with FGS

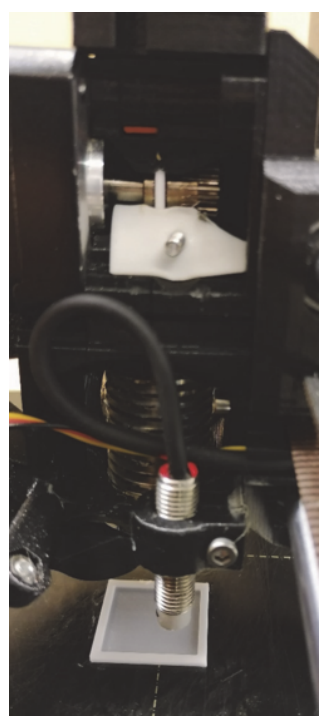

(c) $80 \mathrm{~mm} / \mathrm{s}$ with FGS

Figure 5. Comparison of filament feeding with and without FGS

\subsubsection{Airtightness}

The pressure drop values for floors, roofs and sidewalls are shown in Figures 6, 7 and 8 respectively. Three samples have been printed for each combination of printing speed and wall thickness. For each wall thickness at each selected printing speed, the range of the three measured pressure drop values forms an error bar, and the centers of the four error bars for each wall thickness are connected to show the approximate relationship between pressure drop and printing speed.

In the airtightness test for floors, the thicknesses of at least $0.4 \mathrm{~mm}$ (i.e., 4 layers) are studied, since a floor with the thickness of no more than $0.3 \mathrm{~mm}$ can burst under the air pressure of 1.80 bar. As can been seen from Figure 6, airtightness is good for printing speed below $60 \mathrm{~mm} / \mathrm{s}$. For higher printing speed such as $80 \mathrm{~mm} / \mathrm{s}$, the underextrusion becomes obvious due to insufficient heating of filament by the hot end. It is important to mention that there are also samples with zero pressure drop for the thicknesses of $0.4 \mathrm{~mm}$ and $0.6 \mathrm{~mm}$, which means that high speed printing only raises the probability of air leakage but does not always result in poor airtightness. 


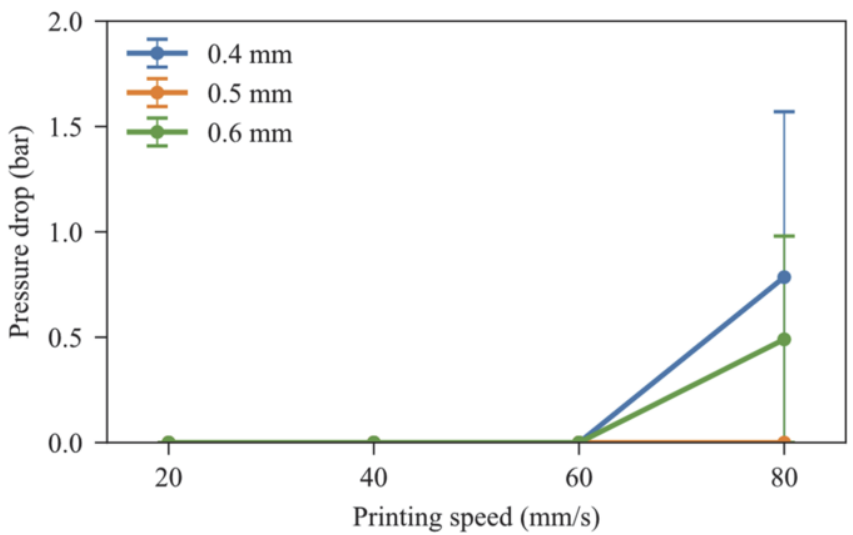

Figure 6. Pressure drop with respect to printing speed for floors

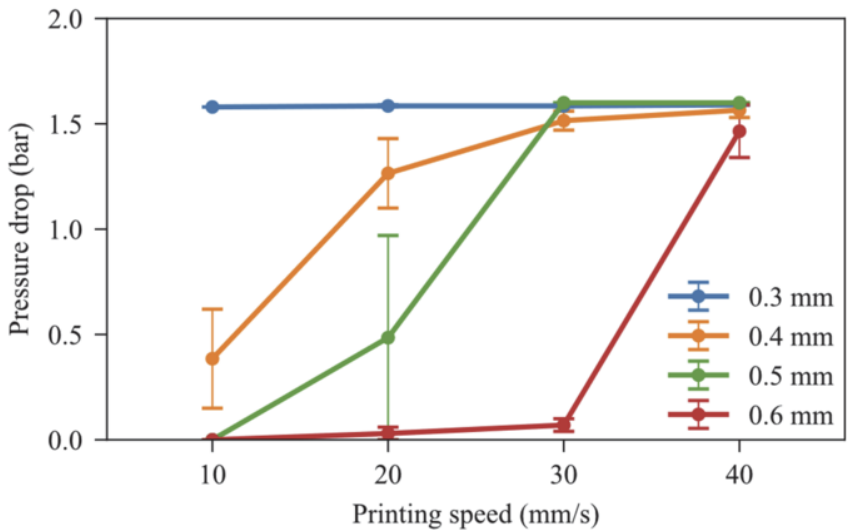

Figure 7. Pressure drop with respect to printing speed for roofs

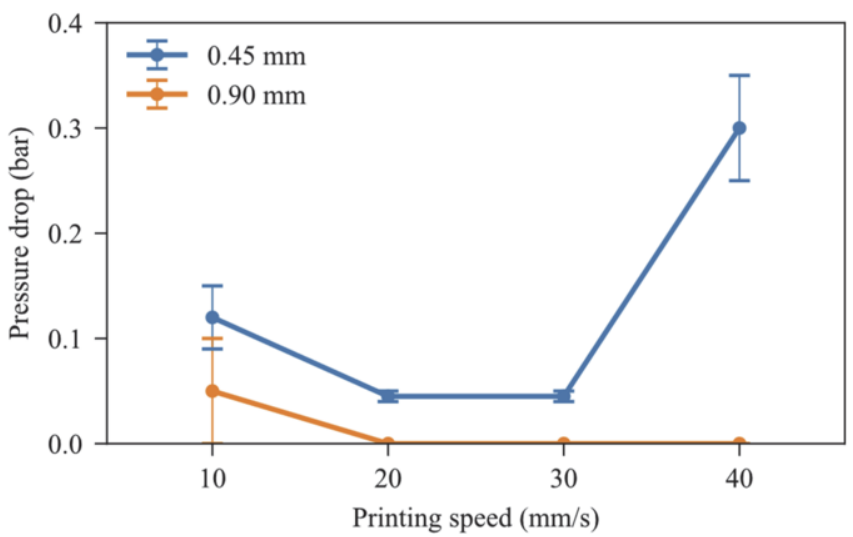

Figure 8. Pressure drop with respect to printing speed for sidewalls 
Figure 7 shows that the pressure drop gradually increases with respect to the printing speed for roofs. By comparing Figure 7 with Figure 6, it can be found that an airtight roof requires lower printing speed and thicker wall than an airtight floor. This is because support structure is not used in printing the roof, and the bottom layers of the roof collapse due to gravity. Tight bonding can be formed above the fifth layer with a low printing speed at $10 \mathrm{~mm} / \mathrm{s}$. An example of the roof wall sample with thickness of $0.4 \mathrm{~mm}$ is given in Figure 9. As the printing speed increases, the under-extrusion of the material becomes more serious and the gap becomes more obvious in the wall, resulting in higher pressure drop and worse airtightness.

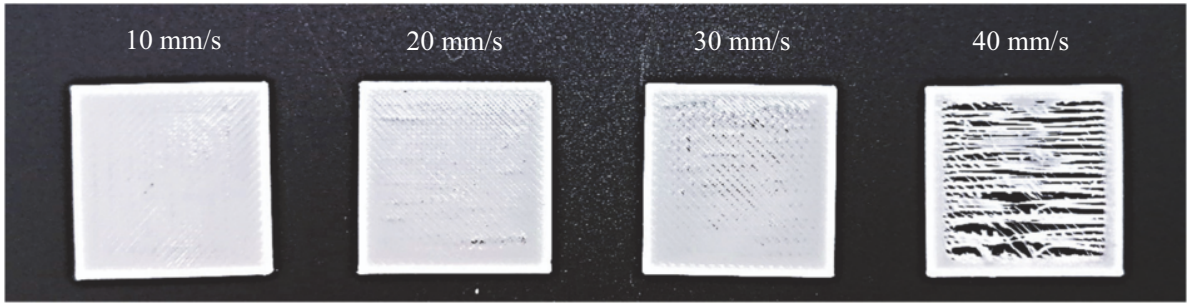

Figure 9. Example of printing quality changing with printing speed (roof with wall thickness of $0.4 \mathrm{~mm}$ )

For the sidewalls, the wall thicknesses of $0.45 \mathrm{~mm}$ and $0.9 \mathrm{~mm}$ have been selected for test since the extrusion width is set to $0.45 \mathrm{~mm}$. If a sidewall is printed with an over-high speed, gaps will be form in the wall due to under-extrusion which is similar to floors and roofs. On the other hand, a low printing speed also contributes to bad airtightness of the sidewall. This may be due to the over-heating of the deposited material in the thin wall which would sink under gravity or be bent by the nozzle and then create gaps between layers.

To conclude, different wall features have different constraints on both the wall thickness and printing speed. In general, thicker wall offers better airtightness and there exists an optimal printing speed for each thickness of each wall feature. For floor, roof and sidewall designed in this paper, at least 4, 5 and 2 layers are recommended respectively when the layer thickness is $0.1 \mathrm{~mm}$, the extrusion width is $0.45 \mathrm{~mm}$ and the air pressure is 1.80 bar.

\section{Conclusion}

In this paper, the extruder structure of the FDM printer has been modified to mitigate the TPU filament buckling problem, and the airtightness with respect to printing speed for three types of wall features in an inflatable product has been studied. The following conclusions have been reached:

1. Adding lateral support to the TPU filament is beneficial for raising the printing speed for TPU material. This is because the axial critical force increases as the unsupported length is reduced by the lateral support. The TPU filament can be printed with a volumetric speed higher than $3.7 \mathrm{~mm}^{3} / \mathrm{s}$ under the support of the proposed filament guide sheet.

2. Different wall features, including floor, roof and sidewall, have different constraints on both the wall thickness and printing speed. Printing of floor is 
least affected by gravity, while printing of roof is most affected by gravity. For the samples in this paper, the recommended minimum numbers of layers for floor, roof and sidewall are 4, 5 and 2 respectively when the layer thickness is $0.1 \mathrm{~mm}$, the extrusion width is $0.45 \mathrm{~mm}$ and the air pressure is $1.80 \mathrm{bar}$.

3. A thicker wall generally has better airtightness than a thin wall and raising the printing speed will also raise the possibility of air leakage for inflatable products due to under-extrusion. For thin sidewalls, there also exists a lower limit for printing speed.

In addition to TPU, the methods proposed in this paper can also be a reference for FDM printing of material with similar or lower hardness than that of TPU. In future research, complex features, such as corners and walls with non-uniform thickness or slope surfaces, as well as thickness change due to wall swelling by inflation will be taken into account. Modelling of TPU material bonding during deposition under various printing speed will be carried out for finding out solutions for printing speed optimisation. Tool path planning and speed optimisation over entire tool path will also be studied.

\section{Acknowledgement}

This research is financially supported by Swedish Research Council (Grant No. 201704550) and KTH XPRES.

\section{References}

[1] W. Dang, E. S. Hosseini, and R. Dahiya, Soft Robotic Finger with Integrated Stretchable Strain Sensor, 2018 IEEE SENSORS, 2018, pp. 1-4.

[2] O. Flamand and J.-C. Thomas, Inflatable walls for wind tunnels, In: 7th European-African Conference on Wind Engineering (EACWE 2017), Liège, 2017.

[3] H.-J. Kim, Y. Tanaka, A. Kawamura, S. Kawamura, and Y. Nishioka, Improvement of position accuracy for inflatable robotic arm using visual feedback control method, In: 2015 IEEE International Conference on Advanced Intelligent Mechatronics (AIM), 2015, pp. 767-772.

[4] J. Nassour, Marionette-based programming of a soft textile inflatable actuator,Sensors and Actuators A: Physical, vol. 291, Jun. 2019, pp. 93-98.

[5] B. Gorissen, D. Reynaerts, S. Konishi, K. Yoshida, J.-W. Kim, and M. D. Volder, Elastic Inflatable Actuators for Soft Robotic Applications,Advanced Materials, vol. 29, no. 43, 2017, pp. 1604977.

[6] M. Zhu, Y. Mori, M. Xie, A. Wada, and S. Kawamura, A 3D printed Two DoF Soft Robotic Finger With Variable Stiffness, In: 2018 12th France-Japan and 10th Europe-Asia Congress on Mechatronics, 2018, pp. 387-391.

[7] Z. Wang, Y. Torigoe, and S. Hirai, A Prestressed Soft Gripper: Design, Modeling, Fabrication, and Tests for Food Handling, IEEE Robotics and Automation Letters, vol. 2, no. 4, Oct. 2017, pp. 19091916.

[8] C. Tawk, A. Gillett, M. in het Panhuis, G. M. Spinks, and G. Alici, A 3D-Printed Omni-Purpose Soft Gripper, IEEE Transactions on Robotics, vol. 35, no. 5, Oct. 2019, pp. 1268-1275.

[9] B. W. K. Ang and C. Yeow, Design and Characterization of a 3D Printed Soft Robotic Wrist Sleeve with 2 DoF for Stroke Rehabilitation, In: 2019 2nd IEEE International Conference on Soft Robotics (RoboSoft), 2019, pp. 577-582.

[10] C. Tawk, G. M. Spinks, M. in het Panhuis, and G. Alici, 3D Printable Vacuum-Powered Soft Linear Actuators, In: 2019 IEEE/ASME International Conference on Advanced Intelligent Mechatronics (AIM), 2019, pp. 50-55.

[11] J. Wang et al., 3D-printed peristaltic microfluidic systems fabricated from thermoplastic elastomer, Microfluid Nanofluid, vol. 21, no. 6, Jun. 2017, pp. 105. 
[12] Y. Yang, P. Zeng, and L. Lei, Numerical simulation study of the T-peel behavior of coated fabric films used in inflatable structures, Journal of Applied Polymer Science, vol. 132, no. 3, 2015.

[13] R. C. Dorf, Ed., The Engineering Handbook, 2 edition, CRC Press, Boca Raton Fla., 2004. 